\title{
Qualidade do Relacionamento Treinador-Atleta e Orientação às Metas como Preditores de Desempenho Esportivo
}

\author{
Francielli Cheuczuk ${ }^{1}$ \\ Luciana Ferreira \\ Patric Paludett Flores \\ Lenamar Fiorese Vieira \\ José Luiz Lopes Vieira \\ José Roberto Andare do Nascimento Junior \\ Universidade Estadual de Maringá
}

\begin{abstract}
RESUMO - Este estudo investigou a qualidade do relacionamento treinador-atleta (RTA) e da orientação às metas (OM) como preditoras para o desempenho de atletas de voleibol. Os participantes $(n=185)$ responderam o Questionário de Relacionamento Treinador-Atleta e o Questionário de Orientação às Metas. Os atletas medalhistas perceberam maior proximidade e comprometimento com o treinador e maior orientação para a tarefa (OT) em comparação aos não medalhistas. O RTA apresentou impacto moderado na OT tanto dos atletas medalhistas (proximidade e complementaridade) quanto das atletas não-medalhistas (compromisso). O compromisso apresentou efeito moderado sobre a orientação para o ego dos nãomedalhistas e das mulheres (não significativo). Conclui-se que quanto mais alto o nível de desempenho das equipes, maior a influência do RTA (complementariedade e proximidade) sobre a OT.
\end{abstract}

Palavras-chave: psicologia do esporte, atletas, voleibol, relacionamento treinador-atleta, objetivos, desempenho

\section{Quality of the Relationship Coach-Athlete and Goal Orientation as Predictors of Sport Performance}

\begin{abstract}
This study investigated the quality of the coach-athlete relationship (CAR) and goal orientation (GO) as predictors of sport performance in volleyball players. Participants $(n=185)$ completed the Coach-Athlete Relationship Questionnaire and the Task and Ego Orientation in Sport Questionnaire. The medalists perceived greater closeness and commitment to the coach and greater task orientation (TO) compared to non medalists. The CAR showed moderate impact on TO among medalists and male athletes (proximity and complementarity) as well as among non-medalists and female athletes (commitment). Commitment showed moderate effect on the ego orientation of non-medalists and women (not significant). It is concluded that the higher the performance level of the teams, the higher the influence of CAR (complementarity and proximity) on TO.
\end{abstract}

Keywords: sport psychology, athletes, volleyball, coach-athlete relationship, goals, performance

A mais importante transição na carreira esportiva de um atleta ocorre da categoria juvenil para a adulta. Nesse período, os atletas estão tipicamente na fase de desenvolvimento de suas carreiras esportivas (Wylleman \& Lavallee, 2004), entretanto trata-se também de um momento em que esses jovens estão cada vez mais propensos ao abandono do esporte competitivo devido às demandas e compromisso do esporte e da escola/universidade (Alfermann \& Stambulova, 2007). Para superar essas demandas do contexto esportivo com sucesso, os jovens atletas contam com diversos recursos pessoais e sociais, e esses recursos são funções do próprio indivíduo, bem como do micro-ambiente social do adolescente (Brandão et al., 2000; Marques \& Samulski, 2009). Na Psicologia do Esporte, a avaliação dos recursos que oferecem suporte aos jovens atletas destina forte ênfase a "tríade esportiva" (atletas, pais e treinadores), com cada componente desempenhando um papel importante para que os participantes respondam com sucesso aos desafios

1 Endereço para correspondência: Departamento de Educação Física, Universidade Estadual de Maringá, Avenida Colombo, 5790, Zona 7, Maringá, PR, Brasil. CEP. 87020-900.E-mail: jllvieira@uem.br colocados diante deles (Davis \& Jowett, 2014; Smoll, Cumming, \& Smith, 2011; Wylleman, De Knop, Sloore, Auweele, \& Ewing, 2002). Nesse contexto, a qualidade do relacionamento treinador-atleta (RTA) tem sido apontada como um importante elemento no desenvolvimento da carreira esportiva do adolescente (Jowett \& Cockerill, 2003; Philippe \& Seiler, 2006) e, consequentemente, um diferencial para o sucesso esportivo (Smoll et al., 2011).

Um dos modelos teóricos que discute a importância da qualidade do RTA é o modelo dos 3 "Cs" (Commitment, Complementarity e Closeness), o qual analisa a qualidade da relação a partir de fatores como os sentimentos, os pensamentos e os comportamentos dos membros de um grupo, verificando se estão mutuamente e causalmente interligados (Kelleys et al., 1983). O modelo é constituído por três dimensões: comprometimento (cognitivo), complementaridade (comportamental) e proximidade (afetivo) (Jowett \& Ntoumanis, 2004). O comprometimento inclui a dedicação, o sacrifício e a satisfação em uma estreita e duradoura relação entre atletas e treinadores. A proximidade reflete o tom emocional que ambos expressam por meio do respeito, da confiança e da apreciação. A complementaridade 
refere-se aos tipos de interação cooperativa existentes, sendo determinante na formação e na manutenção do relacionamento mútuo, avaliando a tarefa e a adaptabilidade (Jowett \& Cockerill, 2003; Yang \& Jowett, 2012).

Estudos recentes têm apontado que o bom RTA tem um grande impacto sobre o desenvolvimento positivo (Jowett \& Cockerill, 2003) da carreira de jovens atletas (Vella, Oades, \& Crowe, 2013), influenciando aspectos psicológicos como a ansiedade (Baker, Cote, \& Hawes, 2000), a motivação (Adie \& Jowett, 2010; Mageau \& Vallerand, 2003), as necessidades básicas (Choi, Cho, \& Huh, 2013), a eficácia coletiva (Hampson \& Jowett, 2012), a satisfação atlética (Jowett \& Nezlek, 2011), a coesão de grupo (Jowett \& Chaundy, 2004), o envolvimento parental (Jowett \& Timson-Katchis, 2005), a paixão esportiva (Lafrenière, Jowett, Vallerand, Donahue \& Lorimer, (2008) e a orientação às metas (Alfermann, Geisler, \& Okade, 2013). Entre essas variáveis, a orientação às metas (OM) tem sido considerada um fator determinante para o sucesso dos atletas em uma competição esportiva (Majzub \& Muhammad, 2010; Rogers \& Spitzmueller, 2009) e durante as diferentes transições da carreira esportiva (Wylleman \& Lavallee, 2004). Assim, o presente estudo pretende enfatizar a qualidade do relacionamento treinador-atleta e da orientação às metas no desempenho esportivo de jovens atletas no contexto do voleibol.

De acordo com a Teoria de Orientação às Metas (Sarrazin, Guillet, \& Cury, 2001), a OM se refere às estruturas de metas situacionais criadas pelos pares sociais, sendo caracterizada a partir de duas distintas orientações: Orientação para o Ego (OE) e Orientação para a Tarefa (OT). A OT refere-se ao esforço, maior persistência e interesse do atleta, o qual tem como parâmetro sua própria referência de rendimento. Dessa forma, o atleta está focado nas habilidades pessoais associadas ao nível de habilidades já adquiridas e não as compara com outros companheiros e/ou adversários (Duda, 1992). A OE está associada a uma maior competitividade, com maior ansiedade durante a competição, avaliando o seu desempenho na comparação com o resultado dos outros para comprovar a sua capacidade (Hall, Kerr, Kozub, \& Finnie, 2007).

Pesquisa recente de Adie e Jowett (2010) no contexto do atletismo universitário britânico revelou que os atletas que perceberam uma relação mais cooperativa, comprometida e de proximidade com seus treinadores eram mais propensos a apresentar uma orientação voltada para a maestria (tarefa) e menos propensos a adotar uma orientação esportiva voltada para o próprio de desempenho (ego) ou de esquiva da responsabilidade. Outro estudo com jovens atletas de natação de diferentes contextos culturais (Alfermann et al., 2013) evidenciou que atletas participantes de contextos com menor pressão externa apresentaram maior OT e melhor percepção de relacionamento com o treinador.

Apesar de tais evidências, pesquisadores ressaltam que ainda existem lacunas a respeito do impacto do RTA sobre a OM de jovens atletas (Adie \& Jowett, 2010; Alfermann et al., 2013). Em primeiro lugar, não se sabe se tal relação se mantém em jovens atletas do sexo masculino e feminino. Em segundo lugar, as evidências encontradas não são totalmente consistentes a respeito da relação entre o RTA e a OM, visto que tais estudos analisaram apenas esportes individuais (natação e atletismo). Assim, mais pesquisas precisam apontar parâmetros sobre a relação entre tais variáveis em outras modalidades no esporte juvenil. Além disso, uma das sugestões apontadas por pesquisadores (Adie \& Jowett, 2010; Jowett \& Nezlek, 2012; Yang \& Jowett, 2013) para futuras investigações é verificar o impacto do RTA sobre as diversas variáveis psicológicas em atletas de esportes coletivos de diferentes níveis competitivos.

Diante disso, em uma tentativa de explorar tal lacuna do conhecimento, o presente estudo teve como objetivo investigar o RTA e a OM em função do desempenho motor de jovens atletas de voleibol, buscando especificamente investigar a qualidade do RTA e da OM como preditoras do desempenho esportivo de jovens atletas do sexo masculino e feminino de equipes de diferentes níveis de desempenho motor (medalhistas e não-medalhistas).

\section{Método}

\section{Participantes}

Fizeram parte do estudo todas as 16 equipes de voleibol participantes do Campeonato Paranaense Sub-18 de 2014, totalizando 185 atletas, sendo 95 do sexo masculino e 90 do sexo feminino, com média de idade de 17,27 $( \pm 1,25)$ anos e com tempo de prática na modalidade de 4,00 $( \pm 2,38)$ anos. A amostra foi dividida em dois grupos (medalhistas e nãomedalhistas) em função da classificação final no Campeonato Paranaense Sub-18 (principal competição para jovens atletas do estado): (a) Grupo 1: Medalhistas - Atletas das equipes que terminaram a competição na $1^{\mathrm{a}}, 2^{\mathrm{a}}$ e $3^{\mathrm{a}}$ posição $(\mathrm{n}=70)$; e (b) Grupo 2: Não-medalhistas - Atletas das equipes que terminaram a competição da $4^{\mathrm{a}}$ posição em diante $(n=115)$.

\section{Instrumentos}

A qualidade do RTA foi mensurada por meio do CoachAthlete Relationship Questionnaire (CART-Q) - Athlete version (Jowett \& Ntoumanis, 2004), validado para o contexto esportivo brasileiro como Questionário de Relacionamento Treinador-Atleta (CART-Q) - Versão Atleta (Vieira et al., 2015). O instrumento consiste de 11 itens distribuídos por três subescalas: Proximidade, Comprometimento e Complementaridade. Os itens são respondidos em uma escala do tipo Likert de 7 pontos, em um continuum de "0" (discordo totalmente) a "7" (concordo totalmente). Para a análise dos dados, médias ponderadas dos resultados dos itens de cada dimensão foram calculadas, inseridas na análise como variáveis observadas. A Análise Fatorial Confirmatória (AFC) apresentou estrutura fatorial satisfatória $\left[X^{2}(36)=69,93 ; X^{2} / g l=2,18 ; C F I=0,94 ; G F I=0,93 ; T L I=0,92\right.$; $R M S E A=0,08]$ e os valores da confiabilidade composta foram adequados (Proximidade $=0,85$; Comprometimento $=0,72$; Complementaridade $=0,83$ ).

Para verificar a OM dos atletas foi utilizado o Task and Ego Orientation Sports Questionnaire (TEOSQ) (Duda, 1989), validado para a língua portuguesa como Questionário 
de Orientação às Metas (Goulart, Rose, \& Rezende, 2007). $\mathrm{O}$ instrumento é composto por 16 itens distribuídos em duas subescalas: Orientação para a Tarefa (OT) e Orientação para o Ego (OE). O questionário é respondido em uma escala Likert de 5 pontos, em um continuum de "0" (discordo totalmente) a "5" (concordo totalmente). Para a análise dos dados, médias ponderadas dos resultados dos itens de cada dimensão foram calculadas, inseridas na análise como variáveis observadas. AFC revelou ajuste aceitável $\left[X^{2}\right.$ $(100)=164,77 ; X^{2} / g l=1,65 ; C F I=0,92 ; G F I=0,90 ; T L I=0,91$; $R M S E A=0,05]$ e confiabilidade composta para a consistência interna foi satisfatória $(O T=0,78 ; O E=0,80)$.

\section{Procedimentos}

A pesquisa está integrada a um projeto institucional sob o parecer do Comitê de Ética em Pesquisa com Seres Humanos (COPEP) $n^{\circ}$ 1.094.824. Inicialmente foi solicitada a autorização para realização da pesquisa junto a Federação Paranaense de Voleibol. Para a coleta de dados, foram contatados os técnicos das equipes participantes do Campeonato Estadual Sub-18 do Paraná de 2014. A coleta dos dados ocorreu no local da competição, após a assinatura do Termo de Consentimento Livre e Esclarecido, com duração média de 35 minutos, no primeiro semestre de 2014.

\section{Análise dos dados}

A análise preliminar dos dados foi realizada por meio do teste de normalidade de Kolmogorov Smirnov. Como os dados não apresentaram distribuição normal, utilizaramse Mediana (Md) e Quartis (Q1-Q3). Para a comparação entre os grupos (medalhistas e não-medalhistas; masculino e feminino), foi utilizado o Teste "U” de Mann-Whitney. Para analisar a relação entre a OM e a qualidade do RTA, utilizou-se a matriz de correlação de Spearman $(p<0,05)$. Tais análises foram conduzidas com o auxílio do software SPSS versão 19.0.

Para verificar a qualidade do RTA e a OM dos atletas, foram conduzidos diferentes modelos de regressão simples uni e multivariada por meio da análise de equações estruturais com as variáveis que obtiveram correlação significativa $(p<0,05)$. A existência de outliers foi avaliada pela distância quadrada de Mahalanobis $\left(D M^{2}\right)$ e a normalidade univariada das variáveis foi avaliada pelos coeficientes de assimetria $(\mathrm{ISkI}<3)$ e curtose $(\mathrm{IKuI}<10)$ uni e multivariada. Como os dados não apresentaram distribuição normal, utilizouse a técnica de Bootstrap de Bollen-Stine para corrigir o valor dos coeficientes estimados pelo método da Máxima Verossimilhança (Marôco, 2010) implementado no software AMOS versão 18.0. Para verificar a adequação da amostra para a análise proposta, aplicamos a técnica de Bootstrapping (MacCallum, Browne, \& Sugawara, 1996). Não foram observados valores de $D M^{2}$ indicadores da existência de outliers nem correlações suficientemente fortes entre as variáveis que indicassem multicolinearidade (Variance Inflation Factors $<5,0)$. Partindo das recomendações de Kline (2010), a interpretação dos coeficientes de regressão teve como referência: pouco efeito $<0,20$, médio efeito para coeficientes até 0,49 e forte efeito $>0,50(p<0,05)$.

\section{Resultados}

Ao comparar a qualidade tanto do RTA quanto da OM em função do sexo, não foi encontrada diferença significativa $(p>0,05)$. No entanto, percebeu-se que tanto os homens quanto as mulheres apresentaram alto nível de qualidade em todas as dimensões do RTA. Ao comparar a qualidade do RTA em função do nível de desempenho motor (Tabela 1), verificou-se diferença significativa nas dimensões de Proximidade ( $p=0,001)$ e Comprometimento $(p=0,001)$, evidenciando que os atletas medalhistas se sentem mais próximos e comprometidos com o treinador em comparação aos não-medalhistas. Em relação à $\mathrm{OM}$, observou-se que os atletas medalhistas demonstraram maior OT $(p=0,016)$. Não houve diferença significativa na $\mathrm{OE}(p=0,938)$.

Ao analisar a correlação entre as dimensões da qualidade do RTA e OM, observou-se que a OT apresentou correlação significativa com todas as subescalas do RTA para os atletas medalhistas e não-medalhistas: Proximidade $(r=0,26 ; r=0,37)$, Compromisso $(r=0,34 ; r=0,38)$ e Complementaridade $(\mathrm{r}=0,51 ; \mathrm{r}=0,39)$. Contudo, a OE apresentou correlação significativa somente com a dimensão de Compromisso $(r=0,29)$ para os atletas não-medalhistas. Para verificar o impacto do RTA sobre a OM em função do

Tabela 1. Comparação da qualidade do RTA e da OM dos jovens atletas de voleibol em função do desempenho na competição

\begin{tabular}{lccc}
\hline \multicolumn{1}{c}{ Variáveis } & $\begin{array}{c}\text { Medalhistas }(\boldsymbol{n}=\mathbf{7 1}) \\
\text { Md (Q1-Q3) }\end{array}$ & $\begin{array}{c}\text { Não medalhistas }(\boldsymbol{n}=\mathbf{1 1 4}) \\
\text { Md (Q1-Q3) }\end{array}$ & $\boldsymbol{p}$ \\
\hline $\begin{array}{l}\text { Relacionamento treinador-atleta } \\
\text { Proximidade }\end{array}$ & $7,00(6,59-7,00)$ & $6,66(5,67-7,00)$ & $0,001^{*}$ \\
Compromisso & $6,00(5,33-6,33)$ & $5,33(4,33-6,00)$ & $0,001^{*}$ \\
Complementaridade & $6,00(5,75-6,50)$ & $6,00(5,25-6,50)$ & 0,761 \\
Orientação às metas & & & $0,016^{*}$ \\
Orientação para a tarefa & $4,00(3,56-4,22)$ & $3,77(3,55-4,00)$ & 0,938 \\
Orientação para o ego & $2,00(1,56-2,78)$ & $2,11(1,63-2,66)$ & \\
\hline
\end{tabular}

Nota. *Diferença significativa: $p<0,05$ 


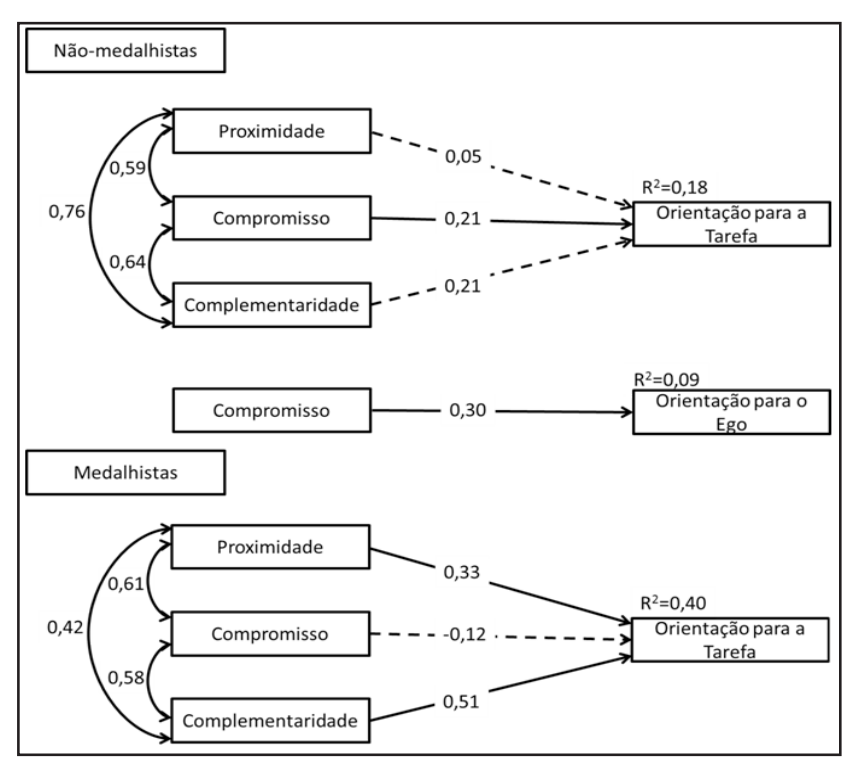

Figura 1. Modelo de regressão do impacto da qualidade do RTA sobre a OM dos atletas medalhistas e não-medalhistas Nota: - - -> trajetória não significativa.

nível de desempenho motor, após a análise de correlação, foi conduzido um modelo de regressão entre as dimensões do RTA e as subescalas da OM que apresentaram correlação significativa para cada grupo $(p<0,05)$.

Verificou-se que (Figura 1) apenas Compromisso apresentou um impacto significativo $(p<0,05)$ na variabilidade da OT $(18 \%)$ dos atletas não-medalhistas. Proximidade e Complementaridade não apresentaram coeficientes de regressão significativos $(p>0,05)$. Além disso, observou-se que Compromisso apresentou impacto significativo $(p<0,05)$ sobre a OE (9\%) para os atletas não-medalhistas. Para os atletas medalhistas (Figura 1), Proximidade e Complementaridade apresentaram impacto significativo $(p<0,05)$ na variabilidade da OT (40\%), enquanto Compromisso não se mostrou significativo $(p>0,05)$.

Em relação às trajetórias individuais do modelo de regressão para os atletas não medalhistas (Tabela 2), verificou-se que o aumento de Compromisso $(\beta=0,21)$ possui um efeito moderado e significativo sobre a OT.

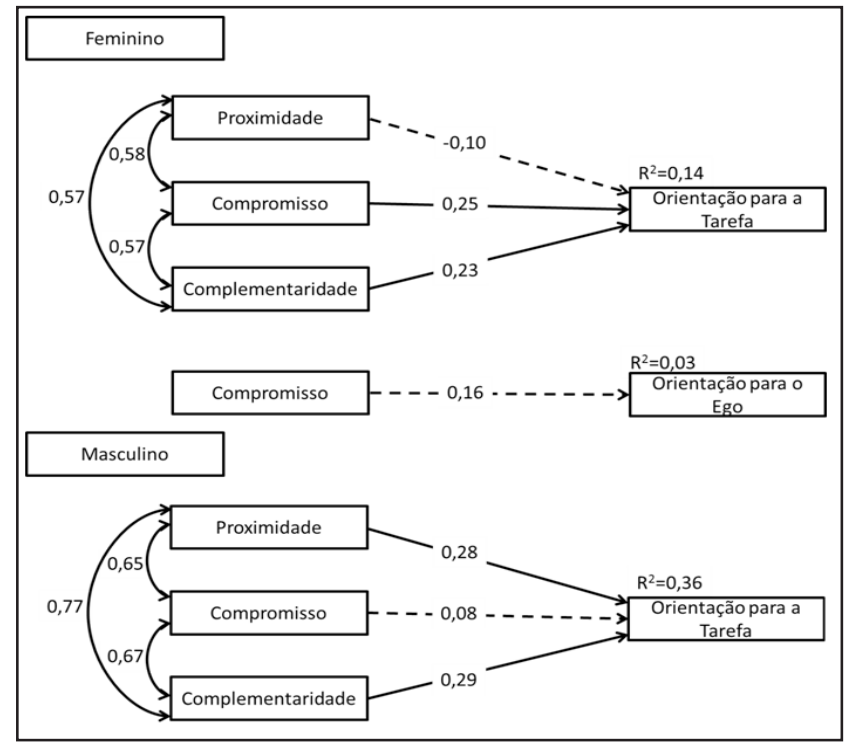

Figura 2. Modelo de regressão do impacto da qualidade do RTA sobre a $\mathrm{OM}$ dos atletas do sexo masculino e feminino Nota: - - -> trajetória não significativa.

Observou-se também que Compromisso demonstrou um efeito moderado sobre a $\mathrm{OE}(\beta=0,30)$. Em relações aos atletas medalhistas (Tabela 2), observou-se que Proximidade $(\beta=0,33)$ e Complementaridade $(\beta=0,51)$ apresentaram um efeito moderado e forte, respectivamente, sobre a OT.

Ao analisar a correlação entre as dimensões da qualidade do RTA e as OM nos atletas de ambos os sexos, observouse que a OT apresentou correlação significativa com todas as subescalas do RTA para os homens e para as mulheres: Proximidade $(r=0,51 ; r=0,21)$, Compromisso $(r=0,46$; $r=0,33)$ e Complementaridade $(r=0,53 ; r=0,33)$. Todavia, a OE apresentou correlação significativa somente com a dimensão de Compromisso $(r=0,32)$ para as mulheres. Para verificar o efeito do RTA sobre a OM em função do sexo, após a análise de correlação, foi conduzido um modelo de regressão entre as dimensões do RTA e da OM que apresentaram correlação significativa $(p<0,05)$ para cada grupo.

Tabela 2. Análise de regressão entre as variáveis com correlação significativa para os atletas medalhistas e nãomedalhistas

\begin{tabular}{cccccccc}
\hline Variável Preditora & Variável Desfecho & $\boldsymbol{r}$ & $\boldsymbol{\beta}$ & $\boldsymbol{R}^{2}$ & $\begin{array}{c}\text { Erro- } \\
\text { Padrão }\end{array}$ & $\begin{array}{c}\text { Rácio } \\
\text { Crítico }\end{array}$ & $P$ \\
\hline Não-medalhistas & & & & & & & \\
Proximidade & & 0,37 & 0,05 & & 0,05 & 0,36 & 0,710 \\
Compromisso & Orientação para a tarefa & 0,38 & 0,21 & 0,18 & 0,03 & 1,89 & $0,048^{*}$ \\
Complementaridade & & 0,39 & 0,21 & & 0,05 & 1,49 & 0,130 \\
Compromisso & Orientação para o ego & 0,29 & 0,30 & 0,09 & 0,05 & 3,36 & $0,001^{*}$ \\
Medalhistas & & & & & & & \\
Proximidade & & 0,26 & 0,33 & & 0,08 & 2,81 & $0,01^{*}$ \\
Complementaridade & Orientação para a tarefa & 0,34 & $-0,12$ & 0,40 & 0,06 & 4,41 & $0,001^{*}$ \\
Compromisso & & 0,51 & 0,51 & & 0,05 & $-0,93$ & 0,34 \\
\hline
\end{tabular}

Nota. *Impacto significativo: $p<0,05$ 
Tabela 3. Análise de regressão entre as variáveis com correlação significativa para os jovens atletas de voleibol

\begin{tabular}{|c|c|c|c|c|c|c|c|}
\hline Variável Preditora & Variável Desfecho & $r$ & $\beta$ & $R^{2}$ & $\begin{array}{l}\text { Erro- } \\
\text { Padrão }\end{array}$ & $\begin{array}{l}\text { Rácio } \\
\text { Crítico }\end{array}$ & $P$ \\
\hline \multicolumn{8}{|l|}{ Feminino } \\
\hline Proximidade & & 0,51 & $-0,10$ & & 0,07 & $-0,73$ & 0,46 \\
\hline Compromisso & Orientação para a tarefa & 0,46 & 0,25 & 0,14 & 0,04 & 1,19 & 0,05 \\
\hline Complementaridade & & 0,53 & 0,23 & & 0,05 & 1,80 & 0,07 \\
\hline $\begin{array}{c}\text { Compromisso } \\
\text { Masculino }\end{array}$ & Orientação para o ego & 0,32 & 0,16 & 0,03 & 0,07 & 1,53 & 0,12 \\
\hline Proximidade & & 0,21 & 0,28 & & 0,06 & 2,08 & 0,03 \\
\hline Complementaridade & Orientação para a tarefa & 0,33 & 0,08 & 0,36 & 0,06 & 2,08 & 0,03 \\
\hline Compromisso & & 0,33 & 0,29 & & 0,03 & 0,70 & 0,47 \\
\hline
\end{tabular}

Nota. ${ }^{*}$ Impacto significativo: $p<0,05$

Verificou-se que apenas Compromisso, apresentou um efeito significativo $(p<0,05)$ na variabilidade da OT $(14 \%)$ das mulheres (Figura 2). Proximidade e Complementaridade não apresentaram coeficientes de regressão significativos $(p>0,05)$. Além disso, observou-se que Compromisso não apresentou efeito significativo $(p>0,05)$ sobre a OE $(3 \%)$ para os atletas do sexo feminino. Para os atletas do sexo masculino (Figura 2), Proximidade e Complementaridade apresentaram efeito significativo $(p<0,05)$ na variabilidade da OT (36\%), enquanto Compromisso não mostrou efeito significativo $(p>0,05)$.

Em relação às trajetórias individuais do modelo de regressão para as atletas do sexo feminino (Tabela 2), verificou-se que o aumento de Compromisso $(\beta=0,25)$ e de Complementaridade $(\beta=0,23)$ possui um efeito moderado sobre a OT. Em relações aos atletas do sexo masculino, observou-se que Proximidade $(\beta=0,28)$ e Complementaridade $(\beta=0,29)$ apresentaram um efeito moderado sobre a orientação para a tarefa.

\section{Discussão}

Embora já existam evidências na literatura a respeito do impacto da qualidade do RTA sobre a OM no contexto do esporte juvenil (Adie \& Jowett, 2010; Alfermann et al., 2013), os achados do presente estudo apresentam significativas contribuições teóricas e práticas para a área da Psicologia do Esporte, visto que analisa a qualidade do RTA e da OM em jovens atletas do sexo masculino e feminino de equipes de voleibol de diferentes níveis de desempenho motor. Dessa forma, os achados desta pesquisa podem servir de parâmetros para treinadores e psicólogos do esporte que trabalham com a formação e o desenvolvimento da carreira esportiva de jovens atletas.

De forma geral, os dados sugerem que Proximidade e Complementaridade apresentaram resultados estatísticos significativos relacionados com a OT dos atletas medalhistas e principalmente do sexo masculino, enquanto Compromisso mostrou efeito significativo na OT dos atletas não-medalhistas e das mulheres. Além disso, verificou-se que Compromisso apresentou efeito sobre a $\mathrm{OE}$ dos atletas não-medalhistas (significativo) e das mulheres (não-significativo). Assim, os resultados do presente estudo sugerem que, para os atletas medalhistas e atletas do sexo masculino, a qualidade do RTA é considerada mais importante para o desenvolvimento da OT em comparação com os atletas não-medalhistas e do sexo feminino.

Ao analisar o modelo de regressão dos resultados dos atletas medalhistas (Figura 1), percebe-se que quanto mais os atletas se percebem próximos e acolhidos pelo treinador, maior o foco na tarefa, evidenciando que atletas bem-sucedidos e que se percebem com boa relação com o treinador estão mais propensos a experimentarem um clima motivacional voltado para a maestria (Adie \& Jowett, 2010; Alfermann et al., 2013). Diversos estudos (Hampson \& Jowett, 2014; Jowett, 2008) ressaltam que, quanto melhor a percepção do RTA, maior é a preocupação do atleta com o desenvolvimento das habilidades pessoais e melhor a união e o comprometimento do grupo na busca das metas da equipe, indicando que as características do RTA exercem um papel preditivo importante sobre a OT no contexto esportivo.

Pesquisas recentes indicam que, quanto melhor a percepção do atleta sobre seu relacionamento afetivo, cognitivo e comportamental com o treinador (Hampson \& Jowett, 2014; Jowett, Shanmugam, \& Caccoullis, 2012), mais o atleta desenvolve uma orientação de clima motivacional com ações voltadas para a busca por novas estratégias voltadas à aprendizagem de novas habilidades, ao invés do foco na superação dos adversários ou até mesmos dos seus companheiros de equipe (Olimpiou, Jowett, \& Duda, 2008). Além disso, essa atitude de busca pela maestria juntamente com a qualidade do RTA favorecem o desenvolvimento de percepção sobre a capacidade do grupo de organizar e realizar tarefas coletivamente e com sucesso (Jowett et al., 2012), corroborando os achados do presente estudo (Figura 1).

Já para os atletas não-medalhistas (Figura 1), observouse que, quanto mais os atletas se percebem comprometidos com o treinador, maior é a OT e a OE, indicando que, para jogadores que ainda não alcançaram o sucesso no esporte, uma relação de comprometimento muito forte com o treinador pode desenvolver o foco tanto na tarefa quanto no ego. Assim, o treinador deve estar sempre atento ao comportamento dos atletas em treinamentos e competições com o intuito de desenvolver um relacionamento eficaz (Jowett \& Cockerill, 
2003), podendo, assim, potencializar o foco nas metas do grupo e não nas metas no desempenho individual.

Percebeu-se também que os atletas medalhistas se perceberam mais próximos e comprometidos com o treinador e com maior OT (Tabela 1), evidenciando que visualizam, no ambiente interpessoal, fortes vínculos pessoais e afetivos de suporte social não apenas em curto prazo, mas também em longo prazo (Davis \& Jowett, 2014), além de serem mais interessados e esforçados em melhorar o desempenho individual e coletivo (Baser, Bayar, \& Ghorbanzadeh, 2013). Tais achados encontram suporte no modelo dos 3 Cs (Jowett \& Ntoumanis, 2004), que ressalta a importância do atleta se sentir próximo e comprometido com seu treinador para o desenvolvimento dos diversos processos psicológicos, como a OM (Alfermann et al., 2013), a eficácia coletiva (Hampson \& Jowett, 2012; Kawazu, Sugiyama, \& Nakasuga, 2012), a coesão de grupo (Jowett \& Chaundy, 2004) e, consequentemente, o sucesso esportivo (Jowett \& Cockerill, 2003).

Ao analisar o modelo de regressão dos resultados dos atletas do sexo masculino e feminino (Figura 2), notase que independente do sexo, o RTA (Compromisso e Complementaridade para as mulheres; Proximidade e Complementaridade para os homens) se mostrou como um fator determinante para a OT. Especificamente, percebese que, quanto mais os homens se percebem próximos e acolhidos pelo treinador, maior o foco na tarefa, evidenciando que as percepções de proximidade e complementaridade são elementos intervenientes para o foco na tarefa. Contudo, nota-se que o comprometimento com o treinador não é um fator significativo para OT dos homens. Já para o sexo feminino (Figura 2), verificou-se que o comprometimento com o treinador pode elevar a tanto a OE quanto a OT. Tais resultados podem ser considerados inéditos na literatura, visto que não existem estudos que analisaram o impacto do RTA sobre a OM isoladamente em atletas do sexo masculino e feminino.

Percebe-se que, para as mulheres, a proximidade com o treinador não se mostrou interveniente na OT (Figura 1), achado que pode estar relacionado a maioria das equipes esportivas, tanto masculinas quanto femininas, serem treinadas por homens, o que pode dificultar a proximidade do treinador com as atletas (Jowett \& Nezlek, 2012). Tal inferência pode ser ilustrada pela entrevista que o técnico da seleção brasileira feminina de voleibol José Roberto Guimarães concedeu recentemente a um programa tele esportivo (Programa tudo em dia, 2014), revelando a dificuldade que teve de se aproximar das atletas quando começou a trabalhar no voleibol feminino porque era muito exigente e não conseguia equilibrar as cobranças por um ótimo desempenho dentro de quadra com as diferentes fases fisiológicas e emocionais que as mulheres passam cotidianamente (tensão pré-menstrual, ciclo menstrual, reações emocionais).

Outro resultado interessante deste estudo foi a correlação significativa entre a dimensão de Compromisso (RTA) com a OE no sexo feminino, indicando que, quando as mulheres se percebem muito comprometidas com o treinador, esse relacionamento pode acabar desenvolvendo um foco no desempenho e sucesso individual (OE). No entanto, quando analisada no modelo de regressão, essa relação não se mostrou significativa (Figura 2).

Além disso, verificou-se que ambos os grupos, tanto do sexo feminino como masculino, apresentaram percepções semelhantes de RTA, OT e OE. Achados de outras pesquisas não confirmam com consistência os resultados sobre o RTA, a OT e a OE entre homens e mulheres, visto que as evidências ainda são escassas. Enquanto alguns estudos apontam que a OE é mais forte nos homens e a OT, nas mulheres (Abraldes, Gómez-López, Granero-Gallegos, \& Rodríguez-Suárez, 2013; Bakirtzoglou \& Ioannou, 2011; Murcia, Gimeno, \& Coll, 2008), outras investigações identificaram que não existe diferença entre os grupos (Hall et al., 2007) ou que OE e OT são mais intervenientes no sexo feminino (Baser et al., 2013).

Apesar das contribuições deste estudo para a literatura, algumas limitações precisam ser analisadas. Primeiro, em relação à abrangência do estudo, que esteve restrito ao estado do Paraná, podendo não representar a população de atletas brasileiros. Contudo, por terem sido as principais equipes do estado na modalidade, que possuem atletas oriundos de diferentes regiões do país e que participam de competições estaduais e nacionais, a amostra pode ser considerada representativa. Outra limitação importante se refere ao caráter transversal do presente estudo, o que não nos permite fazer inferências de causalidade. Talvez um estudo longitudinal fosse capaz de apontar a natureza causal das associações entre as dimensões de RTA e OM. E, por fim, este estudo analisou somente o RTA na versão atleta. Entretanto, seria importante que o RTA fosse avaliado também pelos técnicos das equipes, pois assim teríamos informações efetivas sobre a díade treinador-atleta, comprovando se as percepções que os treinadores possuem do seu relacionamento com os atletas coincidem com as percepções destes.

Diante disso, futuras pesquisas devem analisar o RTA de acordo com a percepção do treinador e o suporte parental desses jovens, construindo assim a "tríade esportiva" (atleta, treinador, pais), bem como acompanhar o resultado dessas equipes durante a temporada, verificando assim a OM nos diferentes momentos de desempenho. Podem ser avaliadas, ainda, outras modalidades (coletivas e individuais) de diferentes regiões do país como também de diferentes categorias e níveis técnicos, visto que a distinção entre os esportes exige da "tríade esportiva" comportamentos diferenciados em relação à comunicação, à proximidade, à confiança e ao compromisso.

Por fim, independente do sexo, destaca-se que a qualidade da relação do treinador com o atleta pode ser considerada uma variável psicológica importante para a execução das tarefas necessárias para o alto desempenho no contexto do esporte coletivo, embora seja maior a magnitude para os atletas medalhistas (vencedores) e do sexo masculino. Ressalta-se que, para os atletas de equipes de menor nível de desempenho motor (não-medalhistas), o comprometimento com o treinador pode desenvolver o foco na tarefa e no ego simultaneamente. Além disso, os atletas com melhor desempenho motor (medalhistas) percebem melhor relacionamento com o treinador e maior orientação para as tarefas ou demandas do contexto esportivo de rendimento, fatores que são considerados determinantes para o sucesso esportivo. 
Sendo assim, algumas implicações práticas podem ser apontadas para os treinadores e psicólogos que trabalham no contexto do voleibol juvenil. Destaca-se principalmente o desenvolvimento de um ambiente interpessoal baseado em suporte social, confiança, comprometimento e proximidade, visto que tal ambiente tende a contribuir para o desenvolvimento do foco na tarefa e nas metas comuns da equipe, favorecendo a melhora da percepção de competência individual e coletiva. Além disso, os achados do presente estudo oferecem novas contribuições a respeito da compreensão e do entendimento da qualidade do RTA e da OM no desempenho esportivo no contexto do voleibol juvenil, destacando a importância do bom RTA para o foco na tarefa dos atletas.

\section{Referências}

Abraldes, J. A., Gómez-López, M., Granero-Gallegos, A., \& Rodríguez-Suárez, N. (2013). The goal orientation of the lifesavers and the relationship with the satisfaction and the beliefs about the causes of success in sport. Cultura Ciencia Deporte, 22(8), 59-66. doi:10.12800/ccd.v8i22.230

Adie, J. W., \& Jowett, S. (2010). Meta-perceptions of the coach-athlete relationship, achievement goals, and intrinsic motivation among sport participants. Journal of Applied Social Psychology, 40(11), 2750-2773. doi:10.1111/j.15591816.2010.00679.x

Alfermann, D., Geisler, G., \& Okade, Y. (2013). Goal orientation, evaluative fear, and perceived coach behavior among competitive youth swimmers in Germany and Japan. Psychology of Sport and Exercise, 14(3), 307-315. doi:10.1016/j. psychsport.2012.11.005

Alfermann, D., \& Stambulova, N. (2007). Career transitions and career termination. In G. Tenenbaum \& R.C. Eklund (Ed.), Handbook of Sport Psychology ( $3^{\mathrm{a}}$ Ed., pp. 712-736). New York: Wiley.

Baker, J., Côté, J., \& Hawes, R. (2000). The relationship between coaching behaviours and sport anxiety in athletes. Journal of Science and Medicine in Sport, 3(2), 110-119. doi:10.1016/ S1440-2440(00)80073-0

Bakirtzoglou, P., \& Ioannou, P. (2011). Goal orientations, motivational climate and dispositional flow in Greek secondary education students participating in physical education lesson: Differences based on gender. Journal Facta Universitatis: Series Physical Education and Sport, 9(3), 295-306.

Baser, B., Bayar, P., \& Ghorbanzadeh, B. (2013). A determination of goal orientation in respect to the age categories and gender volleyball players in relation to their success. European Journal of Experimental Biology - Pelagia Research Library, 3(1), 81-84.

Brandão, M. R. F., Akel, M. C., Andrade, S. A., Guiselini, M. A. N., Martini, L. A., \& Nastás, M. A. (2000). Causas e conseqüências da transição de carreira esportiva: Uma revisão de literatura. Revista Brasileira de Ciência \& Movimento, 8(1), 49-58.

Choi, H., Cho, S., \& Huh, J. (2013). The association between the perceived coach-athlete relationship and athletes' basic psychological needs. Social Behavior and Personality: An International Journal, 41(9), 1547-1556. doi: 10.2224/ sbp.2013.41.9.1547
Davis, L., \& Jowett, S. (2014). Coach-athlete attachment and the quality of the coach-athlete relationship: Implications for athlete's well-being. Journal Sports Science, 32(15), 14541464. doi: 10.1080/02640414.2014.898183

Duda, J. L. (1989). Goal perspectives and behavior in sport and exercise settings. In M. L. Maehr \& C. Ames (Ed.), Advances in motivation and achievement: Motivation enhancing environments (pp. 81-115). Greenwich, CT: JAI Press.

Duda, J. L. (1992). Motivation in sport settings: A goal perspective approach. In G. C. Roberts (Ed.), Motivation in Sport and exercise (pp. 57-91). Illinois: Human Kinetics Books.

Goulart, C., Rose Jr., D., \& Rezende, A. (2007). Tradução e validação do instrumento orientações às metas, aplicado a jovens esportistas brasileiros. Revista de Educação Física, 139, 20-28.

Hall, H. K., Kerr, A.W., Kozub, S.A., \& Finnie, S.B. (2007). Motivational antecedents of obligatory exercise: The influence of achievement goals and multidimensional perfectionism. Psychology Sport Exercise, 8, 297-316. doi:10.1016/j. psychsport.2006.04.007

Hampson, R., \& Jowett, S. (2012). Effects of coach leadership and coach-athlete relationship on collective efficacy. Scandinavian Journal of Medicine \& Science in Sports, 24(2), 454-460. doi: 10.1111/j.1600-0838.2012.01527.x

Jowett, S. (2008). Moderator and mediator effects of the association between the quality of the coach-athlete relationship and athletes' physical self-concept. International Journal of Sports Science Coaching, 2(1),1-20.

Jowett, S., \& Chaundy, V. (2004). An investigation into the impact of coach leadership and coach-athlete relationship on group cohesion. Group Dynamics: Theory, Research, and Practice, 8(4), 302-311. doi: 10.1037/1089-2699.8.4.302

Jowett, S., \& Cockerill, I. M. (2003). Olympic medallists' perspective of the althlete-coach relationship. Psychology of Sport and Exercise, 4(4), 313-331. doi: /10.1016/S14690292(02)00011-0

Jowett, S., \& Nezlek, J. (2012). Relationship interdependence and satisfaction with important outcomes in coach-athlete dyads. Journal of Social and Personal Relationships, 29(3), 287-301. doi: 10.1177/0265407511420980

Jowett, S., \& Ntoumanis, N. (2004). The coach-athlete relationship questionnaire (CART - Q): Development and initial validation. Scandinavian Journal of Medicine \& Science in Sports, 14(4), 245-257. doi: 10.1111/j.1600-0838.2003.00338.x

Jowett, S., Shanmugam, V., \& Caccoulis, S. (2012). Collective efficacy as a mediator of the association between interpersonal relationship and athlete satisfaction in team sports. International Journal of Sport and Exercise Psychology, 10(1), 66-78. doi: 10.1080/1612197X.2012.645127

Jowett, S., \& Timson-Katchis, M. (2005). Social networks in sport: Parental influence on the coach-athlete relationship. The Sport Psychologist, 19, 267-287.

Kawazu, K., Sugiyama, Y., \& Nakasuga, T. (2012). An investigation of the changing relationship between the collective efficacy and team performance of a sport team for different sporting events. Japanese Journal of Sport Psychology, 39(2), 153-167.

Kelley, H. H., Berscheid, E., Christensen, A., Harvey, J. H., Huston, T. L., Levinger, G., . . Peterson, D.R. (1983). Close relationships. New York: Freeman. 
Kline, R. B. (2010). Principles and practice of structural equation modeling ( $3^{\mathrm{a}}$ Ed.). New York: Guilford Press.

Lafrenière, M. K., Jowett, S., Vallerand, R. J., Donahue, E. G., \& Lorimer, R. (2008). Passion in sport: On the quality of the coach-athlete relationship. Journal of Sport and Exercise Psychology, 30, 541-560.

MacCallum, R. C., Browne, M. W., \& Sugawara, H. M. (1996). Power analysis and determination of sample size for covariance structure modeling. Psychological Methods, 1(2), 130-149.

Mageau, G. A., \& Vallerand, R. J. (2003). The coach-athlete relationship: A motivational model. Journal of Sports Sciences, 21(11), 883-904. doi: 10.1080/0264041031000140374

Majzub, R., \& Muhammad, T. A. (2010). Goal orientation, attention styles and anxiety of junior golfers in Malaysia. Procedia Social and Behavioral Sciences, 9, 583-588. doi:10.1016/j. sbspro.2010.12.201

Marôco, J. (2010). Análise de equações estruturais: Fundamentos teóricos, software e aplicações. Pêro Pinheiro: ReportNumber.

Marques, P. M., \& Samulski, D. M. (2009). Análise da carreira esportiva de jovens atletas de futebol na transição da fase amadora para a fase profissional: Escolaridade, iniciação, contexto sócio-familiar e planejamento da carreira. Revista Brasileira de Educação Física e Esporte, 23(2), 103-119. doi: 10.1590/S1807-55092009000200002

Murcia, J. A. M., Gimeno, E. C., \& Coll, D. G. (2008). Relationships among goal orientations, motivational climate and flow in adolescent athletes: Differences by gender. The Spanish Journal of Psychology, 11(1), 181-191.

Olympiou, A., Jowett, S., \& Duda, J. (2008). The psychological interface between the coach-created motivational climate and the coach-athlete relationship in team sports. The Sport Psychologist, 22, 423-438.

Philippe, R. A., \& Seiler, R. (2006). Closeness, co-orientation and complementarity in coach-athlete relationships: What male swimmers say about their male coaches. Psychology of Sport and Exercise, 7, 159-171. doi:10.1016/j. psychsport.2005.08.004
Rogers, A., \& Spitzmueller, C. (2009). Individualism-collectivism and the role of goal orientation in organizational training. International Journal of Training and Development, 13(3), 185-201. doi: 10.1111/j.1468-2419.2009.00326.x

Sarrazin, P., Guillet, E., \& Cury, F. (2001). The effect of coach's task-and ego-involving climate on the changes in perceived competence, relatedness, and autonomy among girl handballers. European Journal of Sport Science, 1, 1-9. doi: 10.1080/17461390100071404

Smoll, F. L., Cumming, S. P., \& Smith, R. E. (2011). Enhancing Coach-Parent Relationships in Youth Sports: Increasing Harmony and Minimizing Hassle. International Journal of Sports Science \& Coaching, 6(1), 13-26.

Vieira, L. F., Nascimento Junior, J. R. A., Pujals C., Jowett, S., Codonhato R., \& Vissoci, J. R. N. (2015). Cross-cultural adaptation and psychometric properties of the Brazilian coachathlete relationship questionnaire (CART-Q) - Athlete Version. Revista Brasileira de Cineantropometria e Desempenho Humano, 17(6):635-649. doi: 10.5007/1980-0037.2015v17n6p635

Vella, S. A., Oades, L. G., \& Crowe, T. P. (2013). The relationship between coach leadership, the coach-athlete relationship, team success, and the positive developmental experiences of adolescent soccer players. Physical Education and Sport Pedagogy, 18(5), 549-561. doi: 10.1080/17408989.2012.726976

Wylleman, P., \& Lavallee, D. (2004). A developmental perspective on transitions faced by athletes. In M. Weiss (Ed.), Developmental sport and exercise psychology: A lifespan perspective (pp. 507-527). Morgantown, WV: Fitness Information Technology.

Wylleman, P., De Knop, P., Sloore, H., Auweele, Y. V., \& Ewing, E. M. (2002). Talented athletes' perceptions of the athlete-coachparents relationships. Kinesiologia Slovenica Scientific Journal on Sport, 8(2), 59-69.

Yang, S. X., \& Jowett, S. (2012). Psychometric properties of the Coach-Athlete Relationship Questionnaire (CART-Q) in seven countries. Psychology of Sport and Exercise, 13(1), 36-43. doi:10.1016/j.psychsport.2011.07.010

Recebido em 13.01.2015

Primeira decisão editorial em 09.02.2016

Versão final em 12.02.2016

Aceito em 12.02.2016 